\title{
Chondromyxoid Fibroma of the Skull Base and Calvarium: Surgical Management and Literature Review
}

\author{
Nasser Khaled Yaghi ${ }^{1}$ Franco DeMonte ${ }^{1}$ \\ ${ }^{1}$ Department of Neurosurgery, The University of Texas M.D. Anderson \\ Cancer Center, Houston, Texas, United States \\ Address for correspondence Franco DeMonte, MD, Department of \\ Neurosurgery-Unit 442, M.D. Anderson Cancer Center, 1515 \\ Holcombe Blvd, Houston, TX 77030, United States \\ J Neurol Surg Rep 2016;77:e23-e34. \\ (e-mail: fdemonte@mdanderson.org).
}

\begin{abstract}
Chondromyxoid fibroma (CMF) is an exceedingly rare tumor that represents less than $1 \%$ of all primary bone neoplasms. Occurrence in the facial and cranial bones is extremely rare and frequently misdiagnosed.

Case Reports We report two cases of CMF, one in the sphenoclival skull base and the other involving the parietal bone in two young female patients. Excision was performed in both cases. Presenting symptoms, treatment, and follow-up are reported.

Methods A retrospective review of the literature on cranial CMF was performed. The location, demographics, presenting symptoms, and treatment of all calvarial and skull base CMF cases published since 1990 are summarized.

Discussion In our literature review, we found 67 published cases of cranial CMF. Mean age of all calvarial and skull base CMFs at diagnosis was 38.2 years old. Of the cases

Keywords

- benign

- bone neoplasms

- cartilage

- calvarium

- skull base affecting the cranium, the sinonasal structures were most commonly involved. To our knowledge we report only the second case of CMF involving the parietal bone published in an English-language journal. Total resection is the best treatment, and should be the goal of surgical intervention. Curettage results in high recurrence rates. Radiotherapy in the setting of subtotal resection or recurrence cannot be definitively recommended and needs further investigation.
\end{abstract}

\section{Introduction}

Chondromyxoid fibroma (CMF) is an exceedingly rare tumor that represents less than $1 \%$ of all primary bone neoplasms. ${ }^{1}$ First described by Jaffe and Lichtenstein in $1948,{ }^{2}$ CMFs need to be distinguished from other aggressive cartilaginous tumors that have significantly different treatments and prognoses. CMF is a benign tumor characterized by lobules of spindle-shaped or stellate cells with abundant myxoid or chondroid intercellular material with a varying number of multinucleated giant cells of different sizes. ${ }^{3}$ Most frequently, it is found in young adults of the second and third decades of life in the lower extremity long bones, particularly arising from the metaphysis. ${ }^{4} \mathrm{CMF}$ can also arise in numerous other anatomic sites. Its occurrence in the facial and cranial bones is extremely rare.

CMF of the cranial bones is an exceedingly challenging diagnosis to make. ${ }^{5,6}$ Zilmer and Dorfman report an initial misdiagnosis rate of $22 \%$ in their series of $36 \mathrm{CMF}$ cases. ${ }^{7}$ Depending on its location, CMF can be difficult to distinguish from an aneurysmal bone cyst, fibrous dysplasia, giant cell tumor, osteoblastoma, osteosarcoma, Ewing sarcoma, mucocele, Langerhans histiocytosis, or even a schwannoma. ${ }^{5,8,9}$ More often, CMF is mistaken for three other myxoid tumors: chordoma, chondroid chordoma, and chondrosarcoma that have a greater frequency of occurrence in the craniofacial skeleton. ${ }^{10,11}$
License terms

Stuttgart · New York

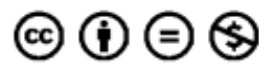

August 15, 2015

accepted

October 21, 2015

published online

January 4, 2016
DOI http://dx.doi.org/

$10.1055 / \mathrm{s}-0035-1570033$. ISSN 2193-6358. 
Here we update the total English literature count to 59 cases of CMF arising from the skull base, including our case of sphenoclival CMF. Additionally, to our knowledge we report the second case of CMF involving the parietal bone published in an English-language journal.

\section{Case 1}

A 38-year-old right-handed woman presented with a 1.5-year history of nasal obstruction and serous rhinorrhea. Approximately 1 month prior to her visit, she also noticed diplopia. A computed tomographic (CT) scan and magnetic resonance imaging (MRI) of the paranasal sinuses were obtained, which showed an expansile lesion of the central skull base, in the sphenoclival region, with extension into the left infratemporal fossa (-Fig. 1). An endoscopic biopsy of the mass was obtained and an initial diagnosis of a malignant fibrohistiocytoma was made. On further review, the pathology was interpreted as showing a "Fibro-mixoid tumor, locally aggressive, and with unknown metastatic potential." This patient's case was discussed at our Joint Planning Conference and the recommended treatment was surgery.

The patient underwent a transmaxillary approach to the anterior cranial fossa with resection of the extradurally located tumor. Postoperative course was uneventful and the patient was discharged on postoperative day 4 .

On follow-up, the patient reported left facial numbness in the V2 distribution and postoperative MRI showed residual tumor at the left lateral portion of the pterygopalatine fissure. The final pathology report was CMF. The minimal residual disease was followed with serial MR imaging.

Over the ensuing year, the patient's tumor showed slow but progressive growth. Surgical resection of the progressive residual sphenoclival CMF was performed. She underwent a left orbitocranial approach to middle cranial fossa with resection of the tumor. Postoperative course was uneventful and the patient was discharged on postoperative day 2 . She is disease free at 4.5 years.

\section{Case 2}

A 31-year-old right-handed woman presented with a 1-year history of brief, mild, left-sided headache. Approximately 3 months prior to her visit, she noticed a lump and tenderness over the left parietal area. As the tenderness increased, she ultimately underwent MR imaging. This revealed the presence of a large lesion centered within the diploe of the parietal bone. There was no intradural

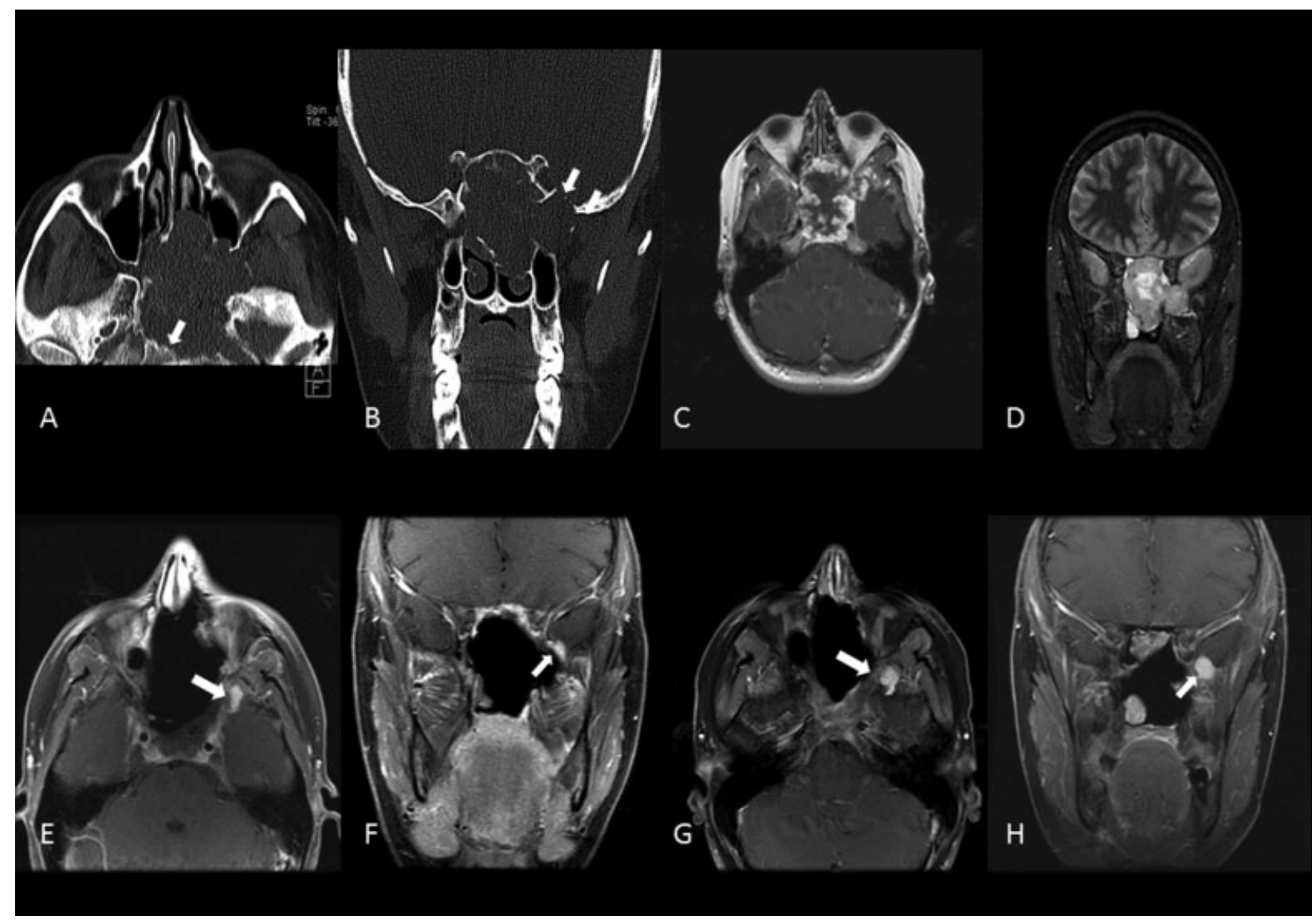

Fig. 1 Case 1. Preoperative axial (B) and coronal (B) CT scans identify this destructive central skull base mass. There is erosion of the clivus (arrow in A) and of the middle fossa floor (arrow in B). Preoperative pos-contrast axial T1-weighted MRI (C) and coronal T2-weighted MRI (D) reveal findings typical for most "chondroid" tumors. Low signal intensity with homogeneous enhancement on T1-weighted postcontrast imaging and high signal intensity on T2-weighted imaging. Images (E) and (F) identify residual disease following the patient first surgery (arrows). Follow-up MRI imaging $(\mathrm{G})$ and $(\mathrm{H})$ reveal progressive enlargement of the residual tumor (arrows) prompting further surgical management. 
extension. At the time of presentation, the patient was in her third trimester of pregnancy, so treatment was postponed to follow her delivery. Subsequent high-resolution CT and MRI images showed a stable tumor, and the patient elected for surgery (-Fig. 2 ).

She underwent resection of the left parietal calvarial lesion and reconstruction with a custom cranial implant. Postoperative course was uneventful and the patient was discharged on postoperative day 3. Her pathology report indicated left parietal calvarial CMF in bone and 3 month follow-up CT showed no evidence of recurrent or residual tumor. She is disease free at 5.5 years.

\section{Methods}

A retrospective literature review was performed. Pubmed search terms included "chondromyxoid fibroma" with English-language filter. Publication dates were limited to January 1, 1990 to April 21, 2013. Search was secondarily refined by article type, case report, and tumor location, calvarial and skull base. Skull base tumors were further categorized into sinonasal, clival/sellar, sphenoid/parasellar, orbit/zygoma, or temporal bone/occiput. All included articles were analyzed and relevant information extracted for the table. Tumor locations and clinical symptoms were standardized for - Table $\mathbf{1}$. This study was conducted with approval from The University of Texas M. D. Anderson Cancer Center Institutional Review Board (IRB) under protocol PA14-0505.

\section{Discussion}

CMF is an exceptionally rare tumor, primarily affecting the long bones and accounting for less than $1 \%$ of all osseous neoplasms. ${ }^{1}$ Its occurrence in the craniofacial skeleton is even less frequent. Wu et al, in their review of 278 cases, reported only 15 tumors involving the skull and facial bones. ${ }^{4}$ The location, demographics, presenting symptoms, and treatment of all of calvarial and skull base CMF cases published since 1990 are summarized in -Table $\mathbf{1}$.

In our literature review, we have found 67 published cases of cranial CMF since 1990. The mean age of all calvarial and skull base CMFs is 38.2 years, which is consistent with the literature. ${ }^{4}$ Patients with clival/sellar and sphenoid/parasellar sites of origin were, on average, a decade older than those with other sites of origin. Ages ranged from newborn to 73 years. Additionally, we found a slightly greater predilection for CMF in females, with 35 females and 24 males. This is conflicting in the literature with some studies reporting a slight male predilection and others a 2:1 female-to-male occurrence. We found a slight male predominance in the temporal bone/occipital site subgroup. Of the cases affecting the cranium, the sinonasal structures were affected most commonly, with the second most common tumor location being the temporal bone and occiput. Most tumors rarely affected a single bone, and therefore appeared to grow without respect to the bony anatomy by involving multiple surrounding bones. Most patients were symptomatic at the time of presentation. The insidious onset of symptoms can

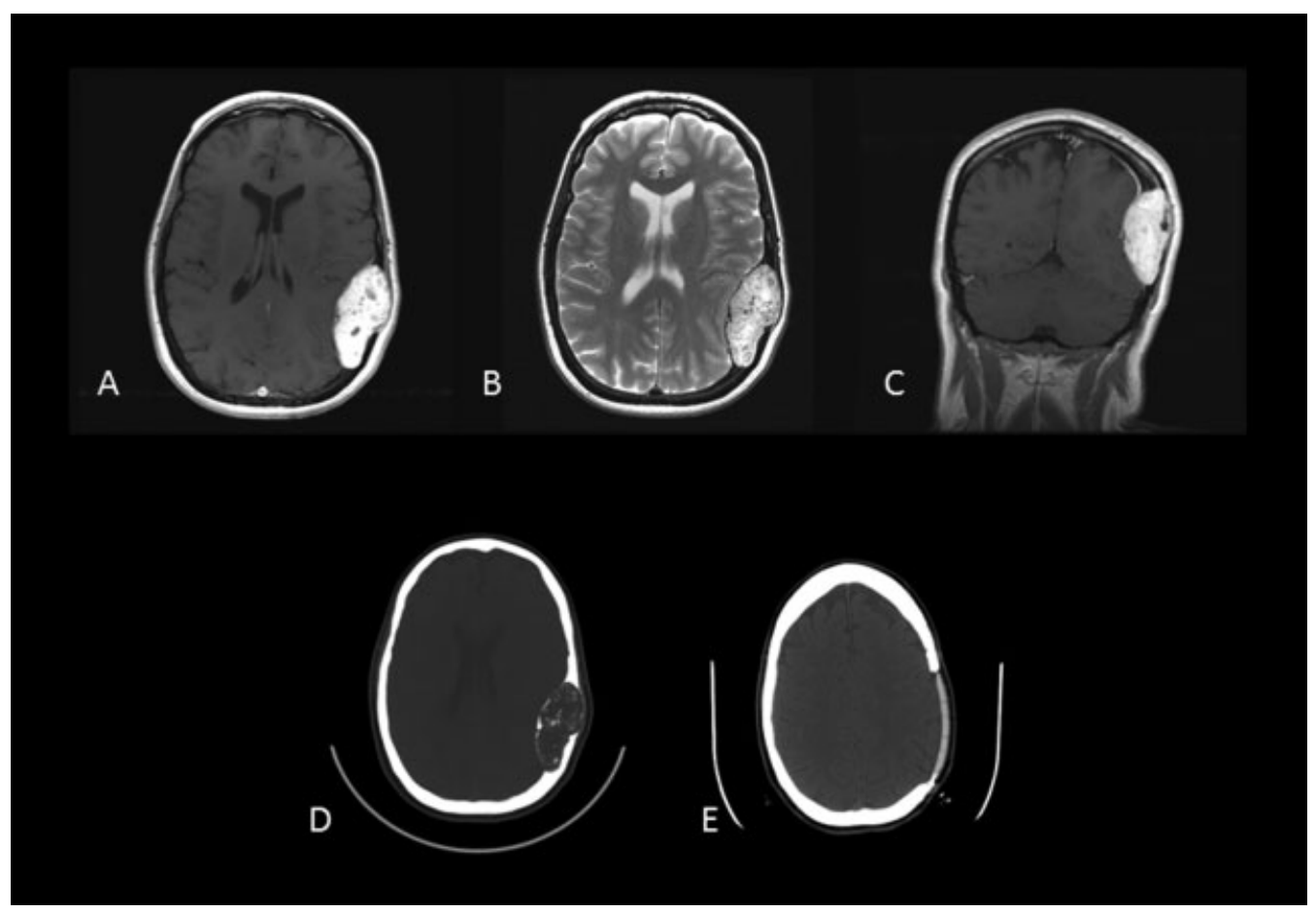

Fig. 2 Case 2. Preoperative axial postcontrast T1-weighted MRI (A), axial T2-weighted MRI (B), and coronal postcontrast T1-weighted MRI reveal a large tumor of the parietal bone with its epicenter in the diploe of the skull. The typical pattern of inhomogeneous enhancement on T1-weighted imaging and hyperintensity on T2-weighted imaging is again seen. Preoperative (D) and postoperative (E) axial CT scans reveal complete tumor removal with custom cranial implant replacing the removed bone. 


\begin{tabular}{|c|c|c|c|c|c|c|c|c|c|c|c|c|c|c|}
\hline & 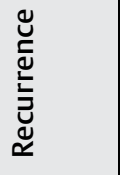 & 1 & 1 & 1 & 1 & 1 & z & in & 2 & & 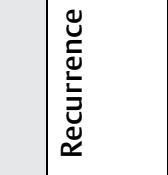 & z) & $\stackrel{\check{\nu}}{\succ}$ & z \\
\hline & 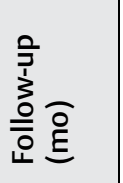 & 1 & 1 & 1 & 1 & 1 & $\stackrel{\operatorname{Ln}}{\sim}$ & $\stackrel{\sim}{i}$ & $m$ & & 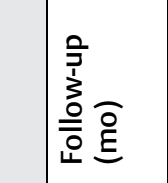 & 0 & 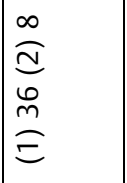 & $\simeq$ \\
\hline & 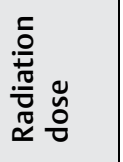 & 0 & 0 & 0 & 0 & 0 & 0 & 0 & 0 & & 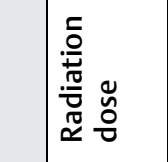 & 0 & 0 & 0 \\
\hline & 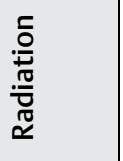 & $\begin{array}{l}0 \\
\text { Oั } \\
z\end{array}$ & $\begin{array}{l}0 \\
\tilde{0} \\
z\end{array}$ & $\begin{array}{l}\stackrel{0}{0} \\
\text { ¿े }\end{array}$ & $\mid \begin{array}{l}0 \\
\tilde{z} \\
2 \\
z\end{array}$ & $\mid \begin{array}{l}0 \\
\tilde{O} \\
z \\
z\end{array}$ & $\begin{array}{l}0 \\
\text { Oे } \\
z\end{array}$ & $\mid \begin{array}{l}0 \\
\vdots \\
0 \\
z\end{array}$ & 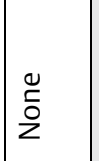 & & 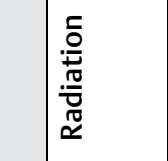 & $\begin{array}{l}0 \\
\tilde{0} \\
z\end{array}$ & $\begin{array}{l}0 \\
\tilde{0} \\
z\end{array}$ & 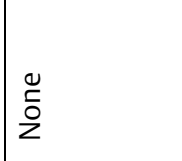 \\
\hline & 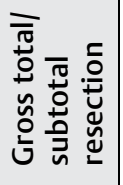 & I & 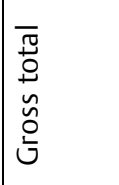 & 1 & 1 & 1 & 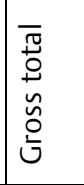 & 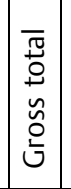 & 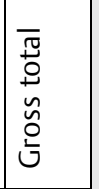 & & 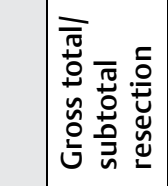 & 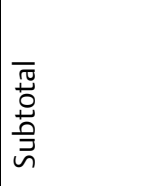 & 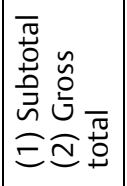 & 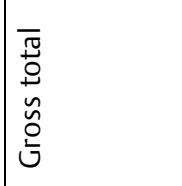 \\
\hline & 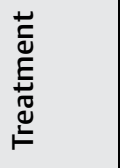 & 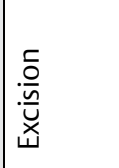 & 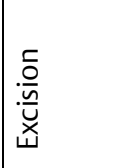 & 1 & 1 & 1 & 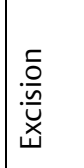 & 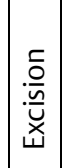 & 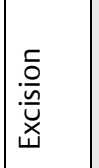 & & 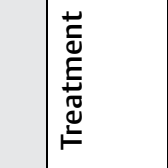 & $\begin{array}{l}. \overline{0} \\
: \frac{0}{\tilde{y}} \\
\dot{x}\end{array}$ & 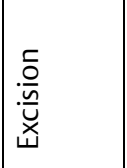 & 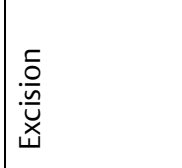 \\
\hline & 䒿 & 1 & 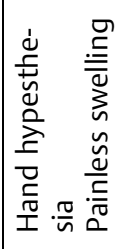 & 1 & 1 & 1 & 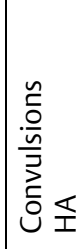 & 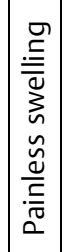 & 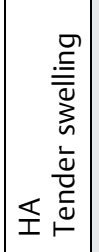 & & 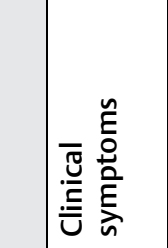 & 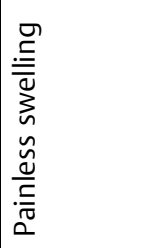 & 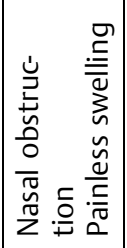 & 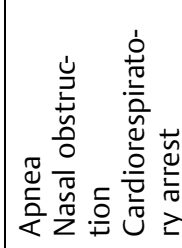 \\
\hline & 次 & 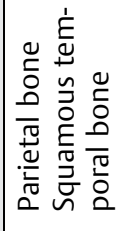 & 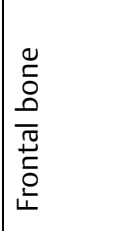 & 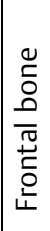 & 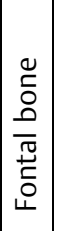 & 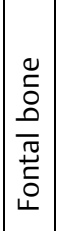 & 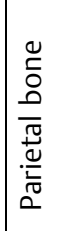 & 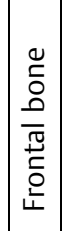 & 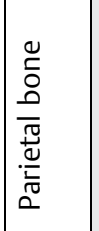 & & 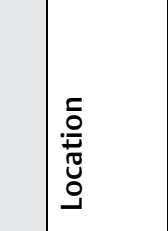 & 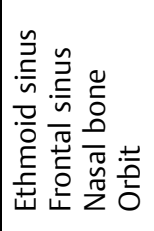 & 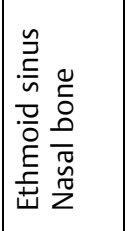 & 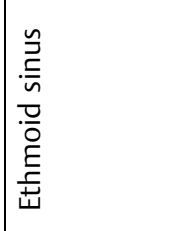 \\
\hline & $\stackrel{\times}{\sim}$ & 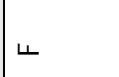 & $\Sigma$ & 1 & 1 & 1 & L & \llcorner & $\sqcup$ & & $\stackrel{\times}{\sim}$ & 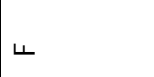 & 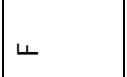 & $\Sigma$ \\
\hline & 苂 & $\underset{\sim}{\sim}$ & $\bar{\sigma}$ & 1 & 1 & 1 & $\mp$ & 导 & $\bar{m}$ & & 荙 & ถิ & $\stackrel{\nabla}{m}$ & \begin{tabular}{|l}
5 \\
0 \\
0 \\
3 \\
3 \\
$z$
\end{tabular} \\
\hline & 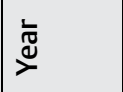 & হ. & مे & $\begin{array}{l}\infty \\
\stackrel{2}{\sigma}\end{array}$ & $\begin{array}{l}\infty \\
2 \\
\swarrow \\
\end{array}$ & $\begin{array}{l}\infty \\
2 \\
\sigma \\
\sigma\end{array}$ & 농 & $\mid \begin{array}{l}0 \\
\stackrel{2}{0} \\
\end{array}$ & $\frac{m}{\grave{N}}$ & & 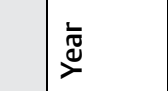 & ํㅗ & ํㅗ & 号 \\
\hline$\Phi$ & 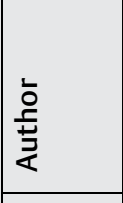 & $\begin{array}{l}\bar{\pi} \\
+ \\
0 \\
0 \\
\frac{n}{3} \\
\overline{3}\end{array}$ & 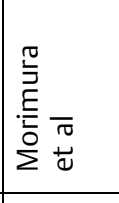 & $\begin{array}{l}\overline{0} \\
+ \\
0 \\
3\end{array}$ & 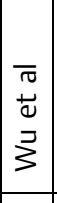 & $\begin{array}{l}\frac{\pi}{\pi} \\
+ \\
0 \\
3 \\
3\end{array}$ & 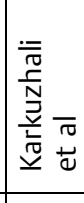 & 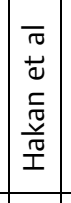 & 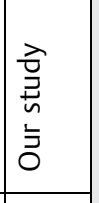 & $\begin{array}{l}\text { 合 } \\
\text { 㟧 }\end{array}$ & 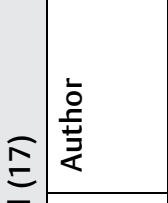 & $\begin{array}{l}\bar{\pi} \\
\stackrel{0}{0} \\
\text { बें } \\
\underline{0}\end{array}$ & 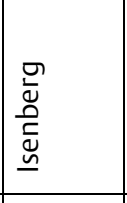 & 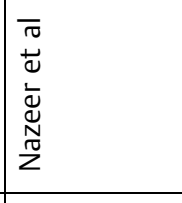 \\
\hline 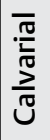 & 崩 & - & $\sim$ & $m$ & $\nabla$ & in & 0 & $\wedge$ & $\infty$ & $\begin{array}{l}\nwarrow \\
己 \\
\vec{د} \\
\text { u }\end{array}$ & 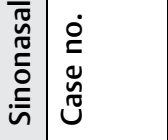 & - & $\sim$ & $m$ \\
\hline
\end{tabular}




\begin{tabular}{|c|c|c|c|c|c|c|c|c|c|c|c|c|c|c|}
\hline & 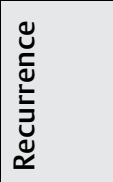 & z & I & $\begin{array}{l}\tilde{x} \\
\tilde{y} \\
\partial\end{array}$ & z & z & 2 & 2 & z & I & I & I & 2) & z \\
\hline & 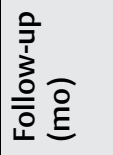 & $\stackrel{\Xi}{\sim}$ & I & 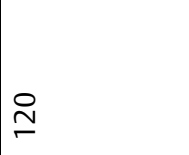 & 0 & $\stackrel{\infty}{\leftarrow}$ & $\approx$ & $\stackrel{\text { m }}{2}$ & $\simeq$ & 1 & 1 & 1 & $\stackrel{\nabla}{\sim}$ & $\stackrel{\sim}{\sim}$ \\
\hline & 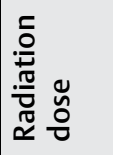 & 0 & 0 & $\begin{array}{l}\text { जे } \\
\text { in }\end{array}$ & 0 & 0 & 0 & 0 & 0 & 0 & 0 & 0 & 0 & 0 \\
\hline & 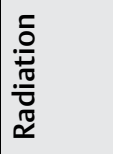 & $\begin{array}{l}\tilde{y} \\
\text { ¿े }\end{array}$ & $\begin{array}{l}0 \\
\text { ò } \\
z\end{array}$ & + & $\begin{array}{l}\stackrel{0}{0} \\
\stackrel{0}{2}\end{array}$ & 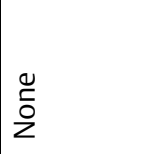 & 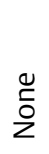 & $\begin{array}{l}0 \\
\tilde{L} \\
z\end{array}$ & $\begin{array}{l}\tilde{u} \\
\tilde{z}\end{array}$ & $\mid \begin{array}{l}0 \\
\tilde{0} \\
2\end{array}$ & 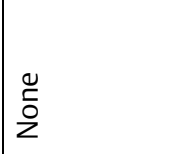 & $\begin{array}{l}0 \\
\tilde{z} \\
z\end{array}$ & $\begin{array}{l}0 \\
\check{2} \\
z\end{array}$ & 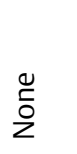 \\
\hline & 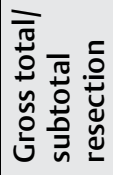 & 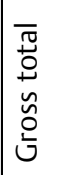 & 1 & 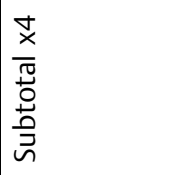 & $\begin{array}{l}\overline{\widetilde{T}} \\
\stackrel{0}{0} \\
\tilde{u} \\
\stackrel{0}{v}\end{array}$ & 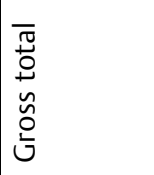 & 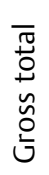 & 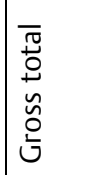 & 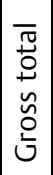 & . & 1 & 1 & 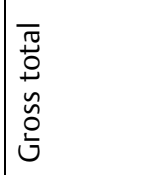 & 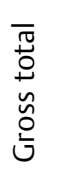 \\
\hline & 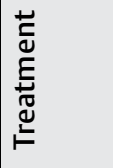 & 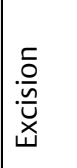 & 1 & 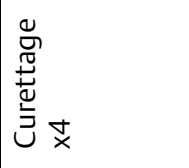 & 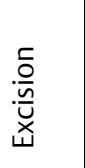 & 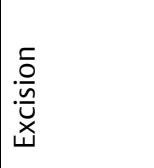 & 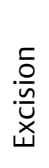 & 蒿 & 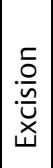 & 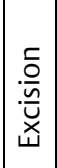 & 1 & I & 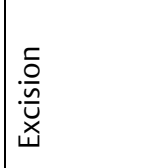 & 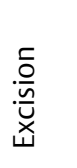 \\
\hline & 䒿 & 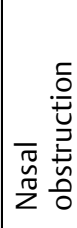 & I & 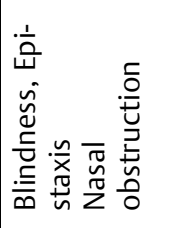 & 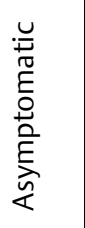 & 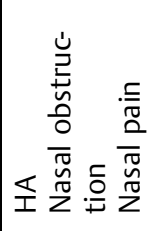 & 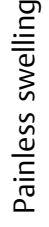 & I & 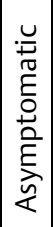 & 1 & 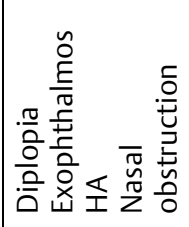 & 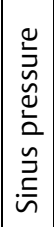 & 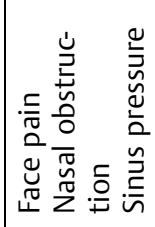 & 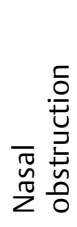 \\
\hline & 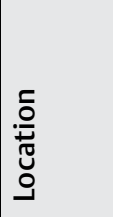 & 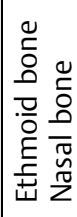 & 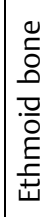 & 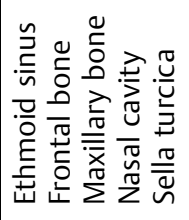 & 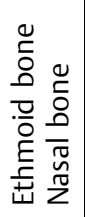 & 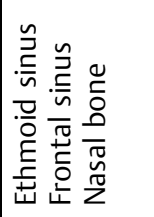 & 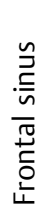 & 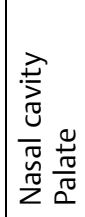 & 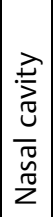 & 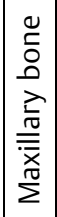 & 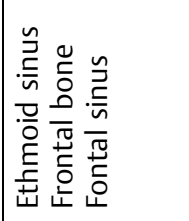 & 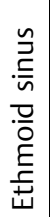 & 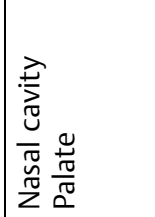 & 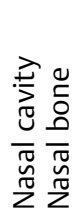 \\
\hline & $\stackrel{\times}{\sim}$ & $\Sigma$ & I & $\leftarrow$ & ч & ч & $\Sigma$ & $\sqcup$ & L & 1 & $\Sigma$ & L & $\Sigma$ & $\Sigma$ \\
\hline & 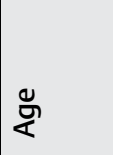 & $\begin{array}{l}5 \\
\vdots \\
0 \\
3 \\
01 \\
z\end{array}$ & I & 0 & 8 & 유 & $q$ & g & 8 & 1 & $\underset{\leftarrow}{\triangleright}$ & $\tilde{q}$ & g & $\begin{array}{l}5 \\
0 \\
0 \\
3 \\
0 \\
z\end{array}$ \\
\hline & $\stackrel{亡}{\check{\varpi}}$ & $\stackrel{\infty}{\mathscr{\sigma}}$ & $\begin{array}{l}\infty \\
\stackrel{2}{\sigma}\end{array}$ & gे & $\underset{\sim}{\stackrel{一}{~}}$ & 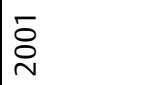 & ڤ̊ㅗㅇ & ¿̊̀ & 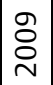 & 㝘 & 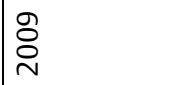 & $\overline{\tilde{N}}$ & $\bar{i}$ & $\stackrel{\sim}{\tilde{N}}$ \\
\hline 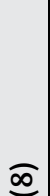 & 高 & 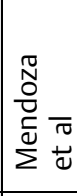 & 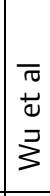 & 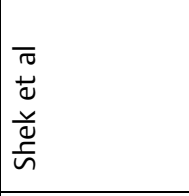 & 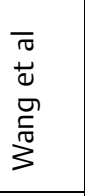 & 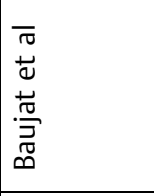 & 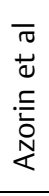 & 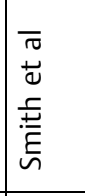 & 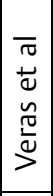 & 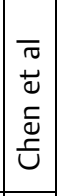 & 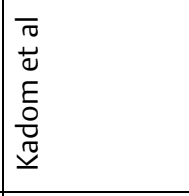 & 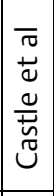 & 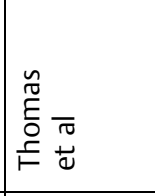 & 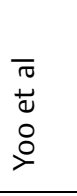 \\
\hline 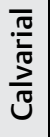 & 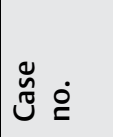 & $\sigma$ & เn & 0 & $r$ & $\infty$ & $a$ & $\stackrel{\circ}{\circ}$ & $=$ & $\simeq$ & $\stackrel{m}{r}$ & $\stackrel{\nabla}{\square}$ & $\stackrel{\operatorname{Ln}}{L}$ & $\ddot{0}$ \\
\hline
\end{tabular}




\begin{tabular}{|c|c|c|c|c|c|c|c|c|c|c|}
\hline & 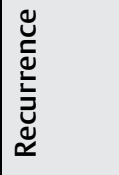 & 2 & & 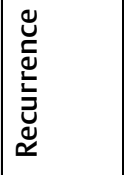 & z & z & z & I & $\begin{array}{l}\tilde{x} \\
\tilde{y} \\
\stackrel{x}{x}\end{array}$ & 2 \\
\hline & 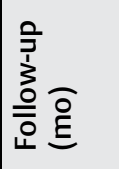 & 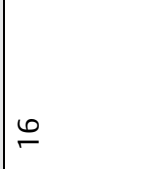 & & 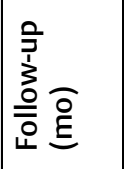 & $m$ & $F$ & $\stackrel{\gtrless}{\sim}$ & 1 & 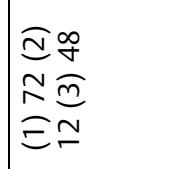 & in \\
\hline & 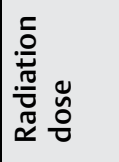 & 0 & & 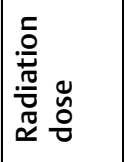 & 0 & $\begin{array}{l}\vec{J} \\
\infty \\
\infty\end{array}$ & 0 & 1 & 1 & 0 \\
\hline & 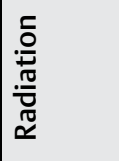 & $\begin{array}{l}\text { Ĩ } \\
\text { z }\end{array}$ & & 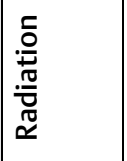 & 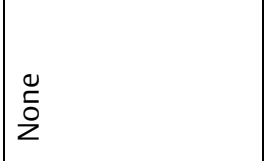 & + & 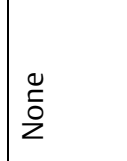 & 1 & $\begin{array}{l}\stackrel{1}{\cong} \\
+ \\
\stackrel{1}{=} \dot{m}\end{array}$ & 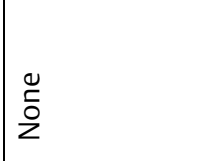 \\
\hline & 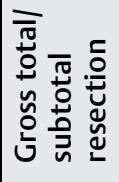 & 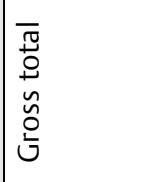 & & 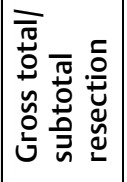 & 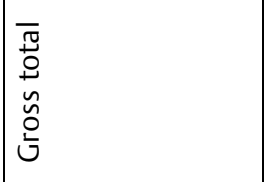 & 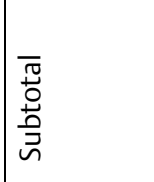 & 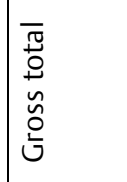 & । & 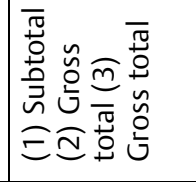 & 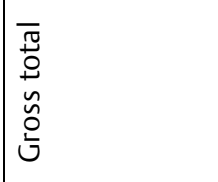 \\
\hline & 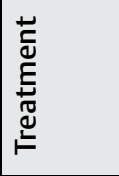 & \begin{tabular}{|l}
.$\overline{0}$ \\
$: \frac{0}{\underline{y}}$ \\
$x$
\end{tabular} & & 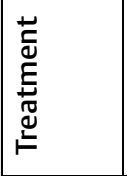 & 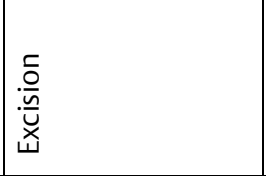 & 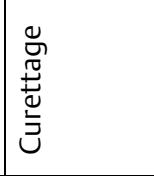 & 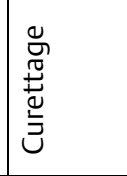 & 1 & 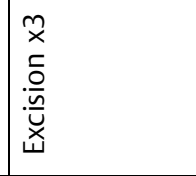 & 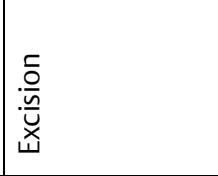 \\
\hline & 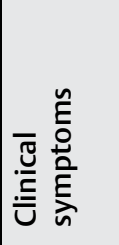 & 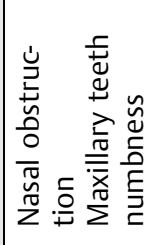 & & ত气 & 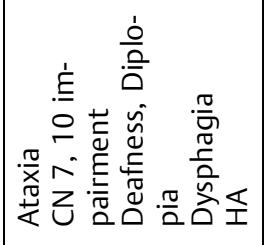 & 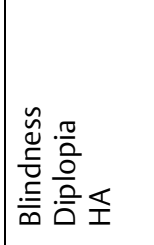 & 袈 & 1 & 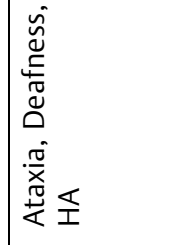 & 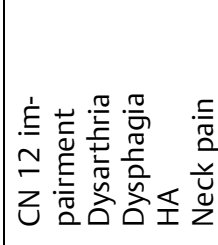 \\
\hline & : & 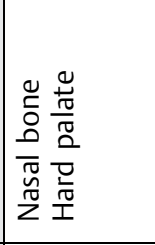 & & 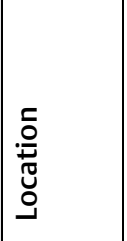 & $\stackrel{n}{\varrho}$ & 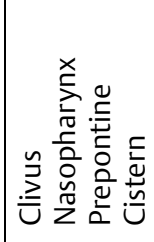 & 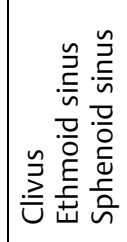 & 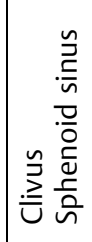 & 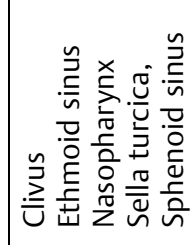 & 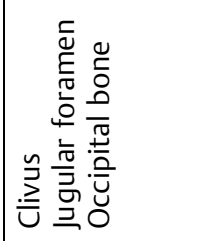 \\
\hline & $\stackrel{\times}{\sim}$ & 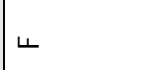 & & $\stackrel{㐅}{\sim}$ & $\Sigma$ & $\sqcup$ & 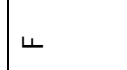 & $\Sigma$ & $\sqcup$ & $\sqcup$ \\
\hline & 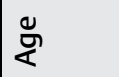 & g & & 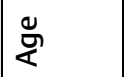 & $\stackrel{m}{r}$ & $\stackrel{+}{m}$ & เి & $\stackrel{\sim}{\sim}$ & ซ & $\stackrel{\infty}{m}$ \\
\hline & 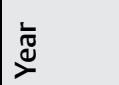 & $\frac{N}{\sim}$ & & 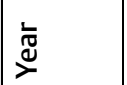 & ஜ & 命 & 合 & 鸟 & $\begin{array}{l}\infty \\
\sigma \\
\sigma\end{array}$ & $\underset{\sim}{\stackrel{D}{~}}$ \\
\hline$\infty$ & 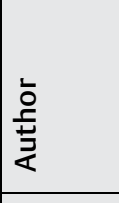 & 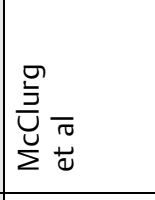 & $\underset{\substack{\sigma \\
\frac{D}{\sigma}}}{ }$ & 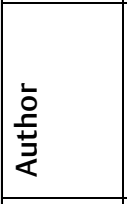 & 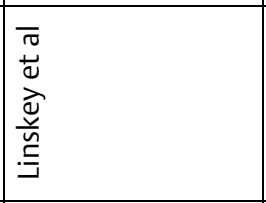 & $\begin{array}{l}\bar{\sigma} \\
+ \\
\bar{\Xi} \\
\underline{\Xi}\end{array}$ & $\begin{array}{l}\bar{\sigma} \\
\tilde{U} \\
\bar{\Xi} \\
\underline{\ddot{U}}\end{array}$ & $\begin{array}{l}\bar{\pi} \\
\stackrel{ \pm}{\square} \\
\bar{\Phi} \\
\underline{\Phi}\end{array}$ & 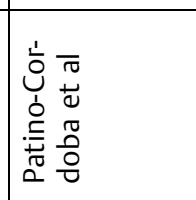 & $\begin{array}{l}\bar{\sigma} \\
\tilde{U} \\
E \\
\vdots \\
\frac{0}{\infty}\end{array}$ \\
\hline $\begin{array}{l}\frac{\bar{\pi}}{\frac{\pi}{\pi}} \\
\frac{2}{\pi} \\
\vec{U}\end{array}$ & 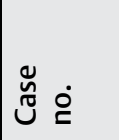 & $\approx$ & 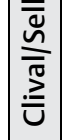 & 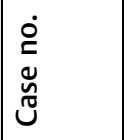 & - & $\sim$ & $m$ & $\nabla$ & in & 0 \\
\hline
\end{tabular}




\begin{tabular}{|c|c|c|c|c|c|c|c|c|c|c|c|c|c|c|c|}
\hline & 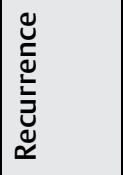 & ì & & 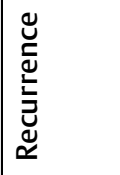 & $\stackrel{\check{\nu}}{\nu}$ & I & $\stackrel{\check{\nu}}{\nu}$ & 1 & 1 & I & $\stackrel{\check{\nu}}{\nu}$ & $\stackrel{\check{\nu}}{\nu}$ & z & 2 & 2 \\
\hline & 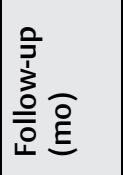 & 6 & & 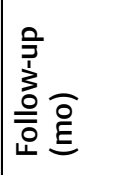 & $\simeq$ & 1 & 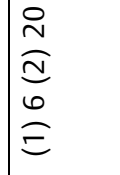 & I & 1 & I & 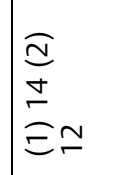 & $\stackrel{\infty}{\sim}$ & 1 & $\stackrel{亠}{\sim}$ & 0 \\
\hline & 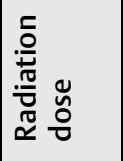 & 0 & & 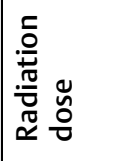 & 0 & 1 & $\frac{3}{6}$ & 0 & 0 & 0 & 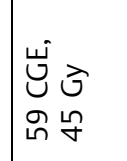 & 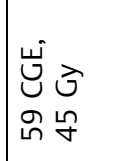 & 0 & 0 & 0 \\
\hline & 竞 & $\begin{array}{l}0 \\
\tilde{0} \\
z\end{array}$ & & 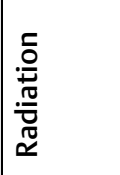 & $\begin{array}{l}\tilde{y} \\
\tilde{0} \\
z\end{array}$ & 1 & 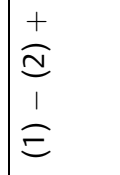 & 童 & 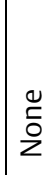 & $\begin{array}{l}\mathscr{y} \\
\check{0} \\
z\end{array}$ & + & + & $\begin{array}{l}0 \\
\check{0} \\
z\end{array}$ & $\begin{array}{l}0 \\
\tilde{z} \\
z \\
z\end{array}$ & 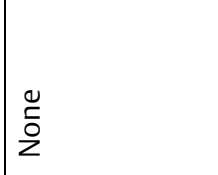 \\
\hline & 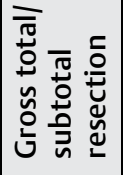 & 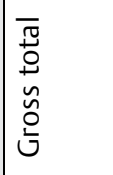 & & 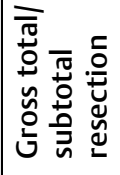 & 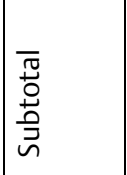 & 1 & 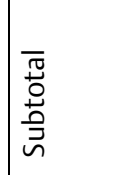 & 1 & 1 & I & 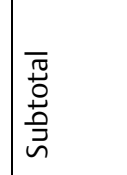 & 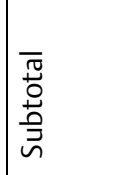 & 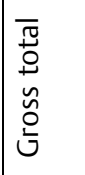 & । & 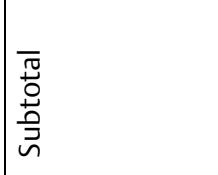 \\
\hline & 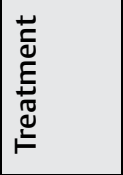 & 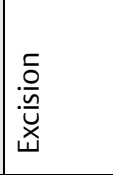 & & 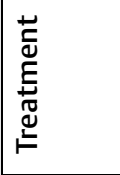 & 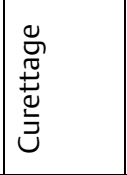 & 1 & 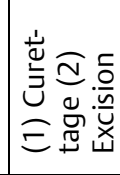 & 1 & 1 & I & 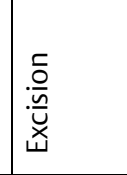 & $\begin{array}{l}\tilde{x} \\
\check{c} \\
. \frac{0}{\underline{y}} \\
\ddot{x} \\
\ddot{y}\end{array}$ & 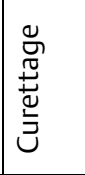 & 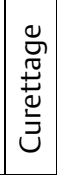 & 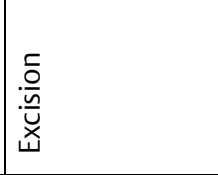 \\
\hline & তֶ气 & 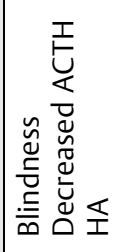 & & 䒿蒿 & 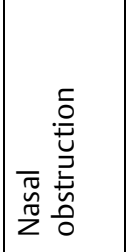 & 1 & 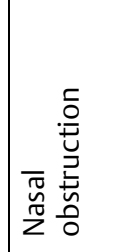 & 1 & 1 & I & $\mid \begin{array}{l}\frac{0}{\bar{a}} \\
\frac{0}{0} \\
\frac{0}{0}\end{array}$ & 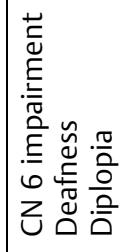 & 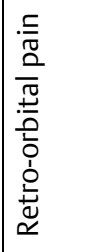 & 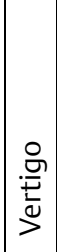 & 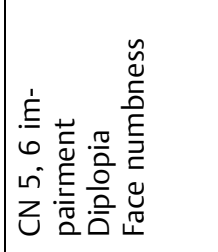 \\
\hline & . & 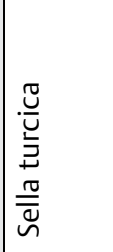 & & . & 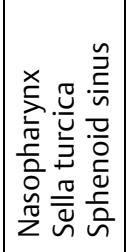 & 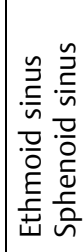 & 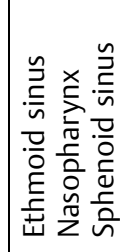 & 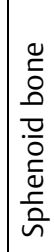 & 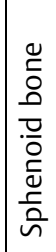 & 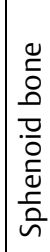 & 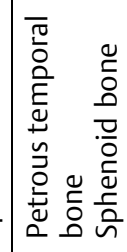 & 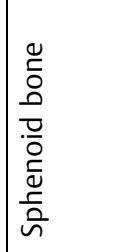 & 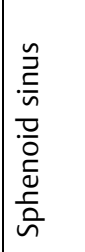 & 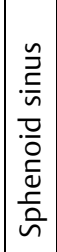 & 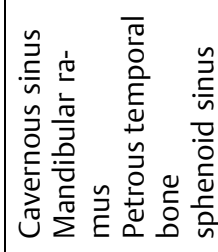 \\
\hline & $\stackrel{\times}{\sim}$ & $\Sigma$ & & $\stackrel{\times}{\sim}$ & 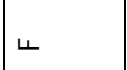 & ᄂ & 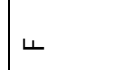 & 1 & 1 & । & $\Sigma$ & $\sqcup$ & $\Sigma$ & L & $\Sigma$ \\
\hline & 荙 & เนn & & 苂 & $\ddot{\theta}$ & ᄃ & $\mathscr{b}$ & 1 & 1 & 1 & 9 & $\stackrel{\infty}{\sim}$ & \& & กี & m \\
\hline & 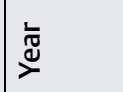 & 六 & & 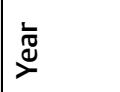 & 吕 & बू & 命 & 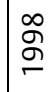 & $\begin{array}{l}\infty \\
\stackrel{2}{\circ} \\
\end{array}$ & જ & 号 & 농 & ஜ & 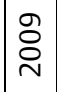 & ஓे \\
\hline ( & 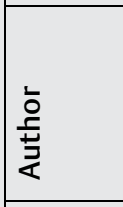 & $\begin{array}{l}\bar{\pi} \\
\stackrel{0}{0} \\
\vec{X}\end{array}$ & 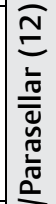 & 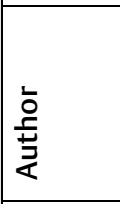 & $\begin{array}{l}\bar{\pi} \\
+ \\
\omega \\
\bar{\Xi} \\
\stackrel{N}{N} \\
\tilde{N}\end{array}$ & 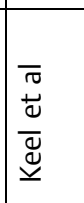 & $\begin{array}{l}\bar{\sigma} \\
\tilde{\omega} \\
\bar{\Phi} \\
\underline{\Xi}\end{array}$ & \begin{tabular}{l}
$\overline{0}$ \\
+ \\
\multirow{2}{3}{}
\end{tabular} & $\begin{array}{l}\pi \\
\pi \\
0 \\
5 \\
3\end{array}$ & $\begin{array}{l}\bar{\pi} \\
+ \\
0 \\
3\end{array}$ & 岕 & 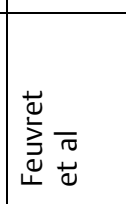 & 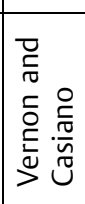 & 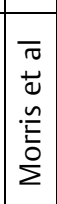 & $\begin{array}{l}\bar{\pi} \\
\overrightarrow{0} \\
\nu\end{array}$ \\
\hline$\frac{\pi}{\frac{\pi}{2}}$ & 芯 & $r$ & $\begin{array}{l}\frac{\overline{0}}{0} \\
\frac{0}{d} \\
\frac{\bar{c}}{n} \\
\text { जे }\end{array}$ & $\begin{array}{l}\dot{\grave{c}} \\
\ddot{y} \\
\tilde{U}\end{array}$ & - & $\sim$ & $m$ & $\nabla$ & Ln & 6 & r & $\infty$ & $a$ & $\circ$ & $\mp$ \\
\hline
\end{tabular}




\begin{tabular}{|c|c|c|c|c|c|c|c|c|c|c|c|c|}
\hline & 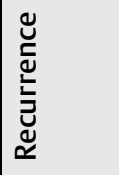 & 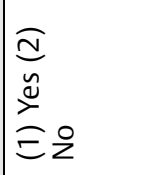 & & 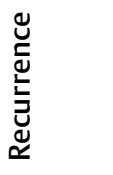 & ㄹ & I & $\stackrel{0}{z}$ & 2 & 2 & 2 & 2 & $\stackrel{\Perp}{\nu}$ \\
\hline & 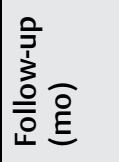 & $\simeq$ & & 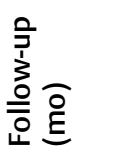 & $\stackrel{\infty}{\sim}$ & 1 & $\stackrel{ \pm}{\sim}$ & $\stackrel{\Xi}{\sim}$ & $\simeq$ & $\stackrel{\Xi}{\sim}$ & ஜ & in \\
\hline & 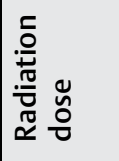 & 0 & & 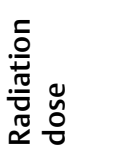 & 0 & 0 & 0 & 0 & 0 & 0 & 0 & 0 \\
\hline & 竞 & 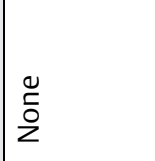 & & & 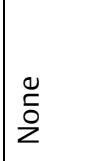 & $\begin{array}{l}0 \\
\tilde{2} \\
2\end{array}$ & 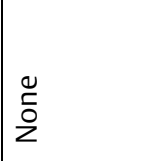 & 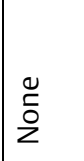 & $\begin{array}{l}0 \\
\vdots \\
\text { Zे }\end{array}$ & 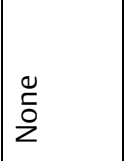 & 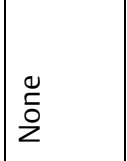 & 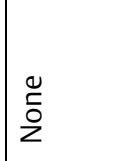 \\
\hline & 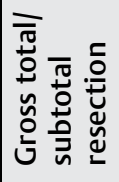 & 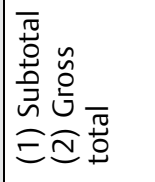 & & 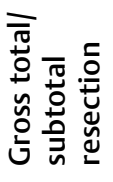 & 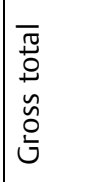 & 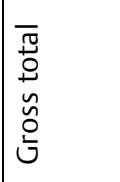 & 1 & 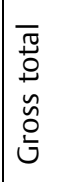 & $\begin{array}{l}\overline{\widetilde{J}} \\
\stackrel{0}{0} \\
\tilde{\hat{O}} \\
\stackrel{0}{0}\end{array}$ & 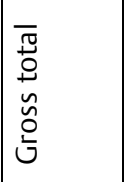 & 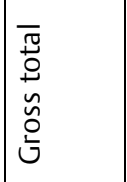 & $\begin{array}{l}\overline{\widetilde{T}} \\
\stackrel{0}{0} \\
\tilde{O} \\
\stackrel{0}{U}\end{array}$ \\
\hline & 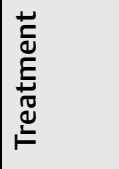 & 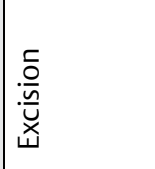 & & 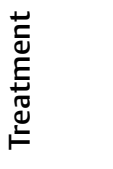 & 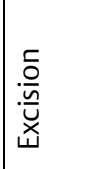 & 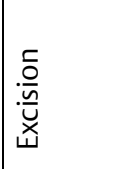 & 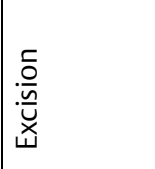 & 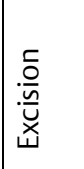 & 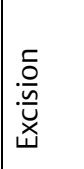 & 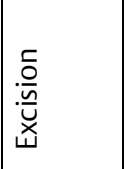 & 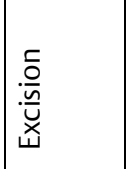 & 言 \\
\hline & তֶ气 & 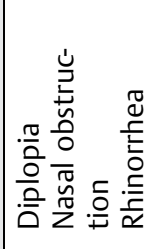 & & 芯产 & 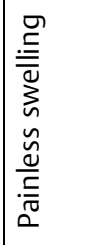 & 至 & 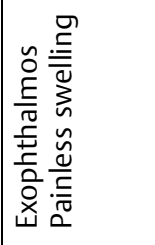 & 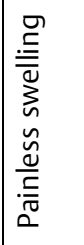 & 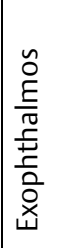 & 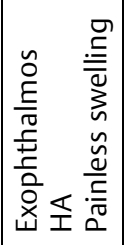 & 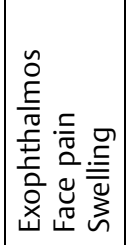 & 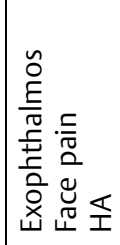 \\
\hline & . & 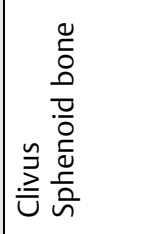 & & 哭 & 泀 & 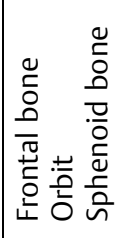 & 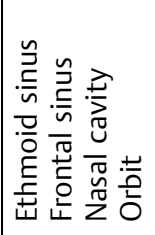 & 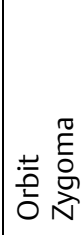 & 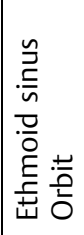 & 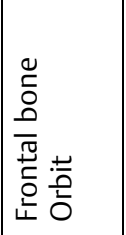 & 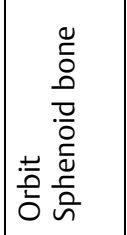 & 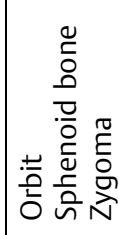 \\
\hline & $\stackrel{\times}{\sim}$ & ப & & $\stackrel{\times}{\sim}$ & $\sqcup$ & ᄂ & $\Sigma$ & $\Sigma$ & L & 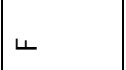 & $\Sigma$ & 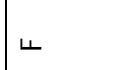 \\
\hline & ğ & $\stackrel{\infty}{m}$ & & 岁 & $\bar{\gamma}$ & $\stackrel{\stackrel{n}{m}}{m}$ & $\approx$ & เn & $\circ$ & $\hat{m}$ & $\stackrel{\nabla}{\sim}$ & n \\
\hline & 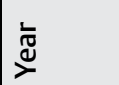 & $\frac{m}{2}$ & & 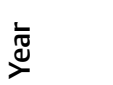 & مू & S̆ & $\begin{array}{l}\infty \\
\stackrel{\circ}{\sigma}\end{array}$ & ஜ̊ & 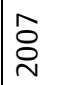 & ஓ्̀ & $\stackrel{\sim}{\stackrel{N}{\sim}}$ & $\stackrel{\sim}{\stackrel{N}{N}}$ \\
\hline$\infty$ & 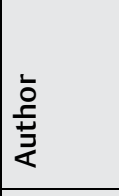 & 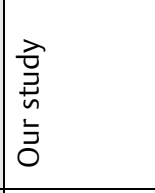 & 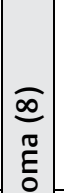 & 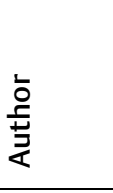 & 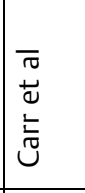 & $\begin{array}{l}\pi \\
\pi \\
4 \\
4 \\
\frac{\pi}{0} \\
3\end{array}$ & 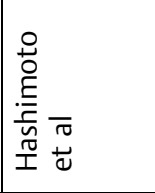 & 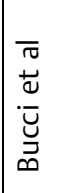 & 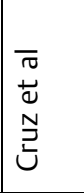 & 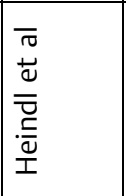 & 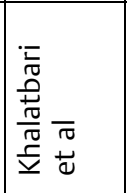 & 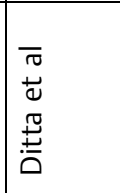 \\
\hline 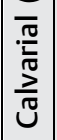 & $\ddot{n}$ & $\stackrel{\sim}{\sim}$ & $\begin{array}{l}\frac{1}{3} \\
\frac{1}{ \pm} \\
\frac{ \pm}{0} \\
0\end{array}$ & $\begin{array}{l}\dot{\circ} \\
\dot{\Xi} \\
\tilde{U}\end{array}$ & - & $N$ & $m$ & $\nabla$ & in & 0 & $r$ & $\infty$ \\
\hline
\end{tabular}




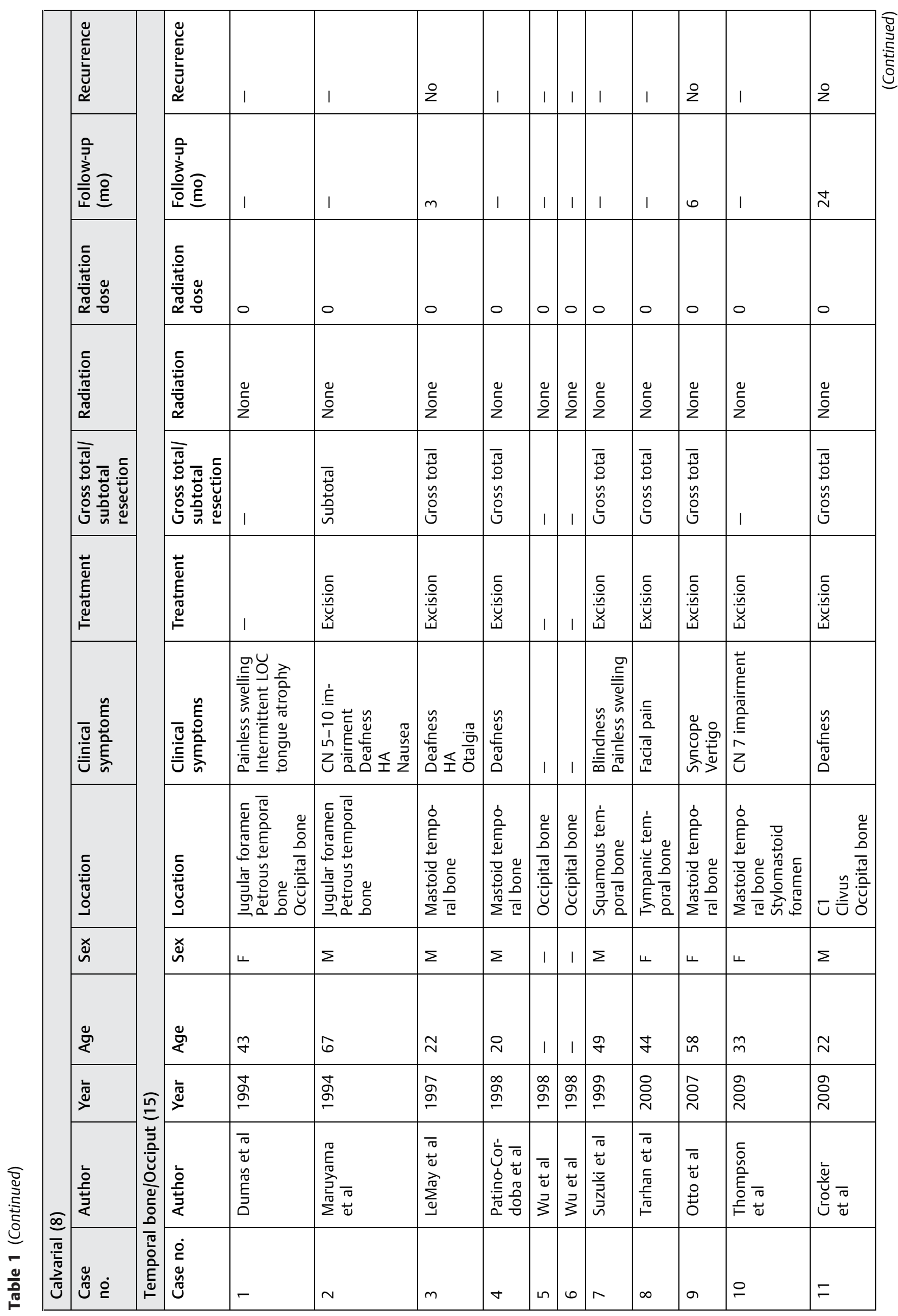


e32 Surgical Management and Literature Review of CMF Yaghi and DeMote

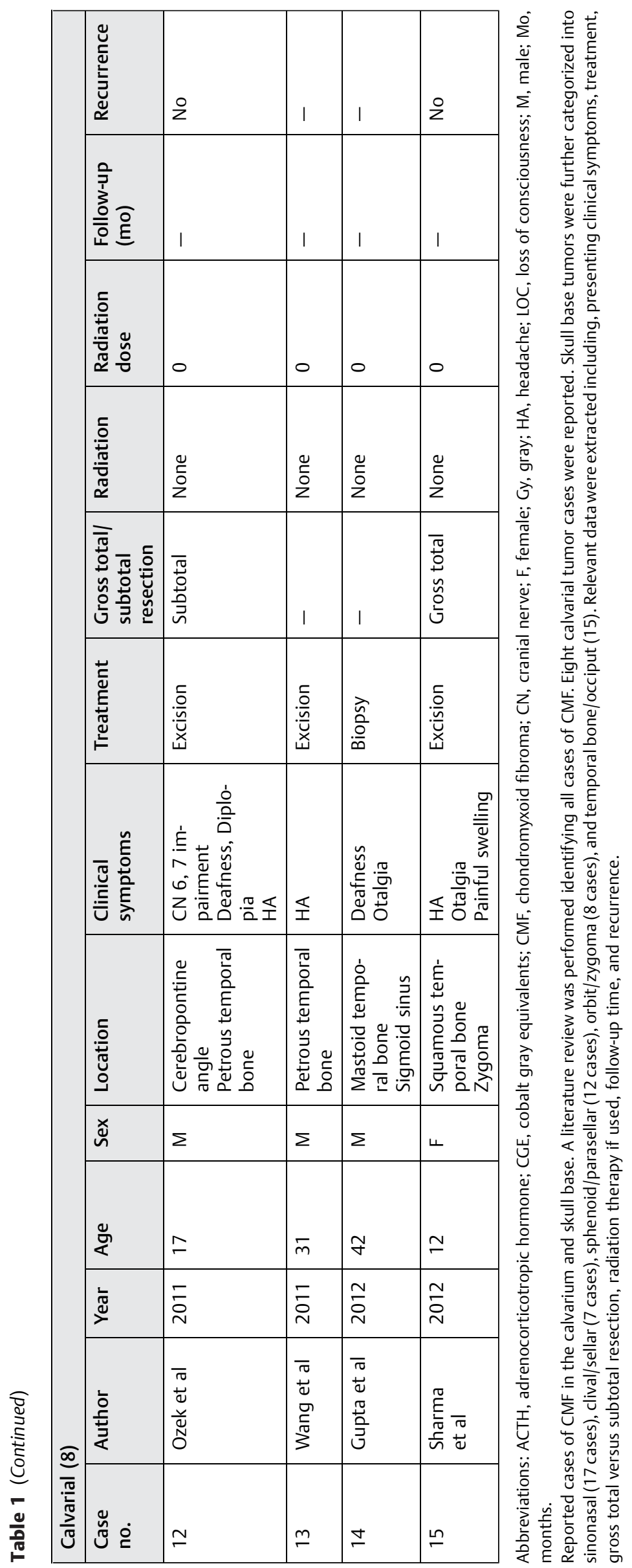

Journal of Neurological Surgery Reports Vol. 77 No. R1/2016 
result in a potentially delayed diagnosis for skull base lesions. ${ }^{12}$ Symptoms included painless or tender swelling, headache, nasal obstruction, exophthalmos, diplopia, deafness, and otalgia. The most common presenting symptom for calvarial CMF was swelling. For the skull base lesions, the most common presenting symptoms appeared to be related to their respective locations. For sinonasal CMF, the most common presentation was nasal obstruction, whereas clival/ sellar lesions presented most commonly with headache. Sphenoid/Parasellar lesions presented with diplopia and orbital/zygomatic CMF presented most commonly with exophthalmos. Last, temporal bone/occipital CMF was most commonly associated with deafness at presentation.

Given this tumor's rare propensity for the calvarium and skull base, we also reviewed our own institution's experience with CMF. Since 1957 to present, MDACC has seen 36 patients with CMF. Of these patients, there were two cases affecting the spine: one at $\mathrm{C} 2$ and the other at S1/ilium. There were only one calvarial case and one skull base case, ${ }^{13}$ both reported here.

Radiologic findings are not diagnostic, but they can offer insight into the diagnosis before intervention. Classically CMF is described as a "radiolucent, lobulated, circumscribed lesion with a sclerotic rim and cortical expansion or erosion" 14 and calcification is rare. Because of the low occurrence rate of this tumor, MRI findings have not been clearly established. However, similar to other bone tumors, CMF has low signal on T1-weighted and high signal on T2-weighted images owing to its cartilaginous nature. ${ }^{14}$ The most challenging aspect to radiologic diagnosis of CMF is the high variability of involved sites. Therefore suspicion for CMF should be maintained when evaluating solitary bone lesions.

Histopathologic analysis of CMF reveals a myxoid lesion containing a paucicellular center, bland stroma cells, reactive boney spicules, and hyaline cartilage with up to $75 \%$ of lesions in the skull and facial bones also containing matrix calcifications. $^{8,15}$ Though not always present, the characteristic features of CMF include lobular appearance, chondromyxoid stroma, and fibrous tissue with multinucleated giant cells. ${ }^{16}$ Nielsen et al performed ultrastructural examination on six tumor samples finding populations of cells with features of three different cell types: chondrocytes, myofibroblasts, and a mixture of both chondrocytes and myofibroblasts. ${ }^{17}$ It should be noted that if the lesion shows significant atypia or mitotic activity, the diagnosis of CMF should be reconsidered. CMF is most commonly found to be positive for vimentin, smooth muscle actin, desmin, S-100 (variably), and CD34. ${ }^{18}$ Generally CMF is negative for pancytokeratin, carcinoembryonic antigen (CEA), and GFAP, and also has a low proliferation rate visualized by $\mathrm{K}_{\mathrm{i}}-67$ staining. ${ }^{19}$ Veras et al describe SOX9 staining in a case of sinonasal CMF. SOX9 has been previously described as a chondrogenesis "master regulator" and plays a role in early-phase chondrocyte differentiation. ${ }^{15}$

Chondrosarcoma, chondroblastic osteosarcoma, fibrous dysplasia, chondroblastoma, and chordoma are included in the differential diagnosis. ${ }^{20,21}$ This is an important distinction to make given the relatively benign nature of CMF. Low-grade chondrosarcomas and CMF share stellate cells, S-100 protein stain positivity, and negative keratin stain. However, chondrosarcomas are more infiltrative than CMFs, which tend to "push" adjacent bony trabeculae. ${ }^{10}$ Additionally, chondrosarcomas lack the fibrous component seen in $\mathrm{CMF}^{22}$ Desai et al, in their study of $10 \mathrm{CMF}$ cases, reviewed the characteristics of the histologic differential diagnosis. ${ }^{20}$ Chondroblastic osteosarcoma diagnostically has osteoid production, of which CMF does not, but it may be difficult to differentiate when there are large patches of CMF-like tissue present. Fibrous dysplasia has a myxoid appearance, but it is differentiated from CMF by its irregular osteoid seams. Chondroblastoma has prominent calcifications and an eosinophilic polygonal cytoplasm that is differentiated from the stellate cytoplasm of CMF. Last, chordoma tends to have large tumor cells, epithelioid with eosinophilic or vacuolated cytoplasm arranged in nests or cords. $^{22}$ Additionally, chordoma stains positive for S-100, epithelial membrane antigen (EMA), and cytokeratins whereas CMF is only positive for $\mathrm{S}-100 .^{22}$

Though it had previously been thought that CMF is an acquired lesion, there is evidence suggesting a possible genetic link. Though cytogenetic studies are limited, Smith et al report there are 14 cases of CMF in the literature with known karyotypes. Notably 11 of the 14 cases had nonrandom clonal abnormalities of chromosome 6 . In particular, rearrangements of the chromosome 6 long arm were most frequent, with four of the cases having pericentric inversion inv(6)(p25q13). ${ }^{23}$ Importantly, chromosome 6 has been implicated in normal cartilaginous development, carrying genes BMP6 (bone morphogenetic protein 6), COL9A1 (collagen type $9 \alpha 1$ ), COL10A1 (collagen type $10 \alpha 1$ ), and IGF2 (insulin-like growth factor 2). Additionally, supporting the possible genetic propensity for CMF, our literature review revealed three cases of congenital CMF presenting in newborns.

Recommended first-line treatment is surgical resection. ${ }^{6}$ Given the benign nature of CMF, surgery can provide a cure. In our literature review, most patients received excision. Of the 67 cases reported here, 43 had excision, 6 curettage, and 2 received both. Two patients received only biopsy, and 14 cases did not report treatment. Of all the cases included here, there were nine recurrences. All but one case of recurrence was associated with subtotal resection, five with excision and three with curettage. Only one recurrence occurred in the setting of gross total resection in which the patient had the extraosseous component excised, while the bone itself was curetted until all visible tumor removed. Twenty-five cases had no data on recurrence.

Efficacy and need for radiotherapy is controversial. Some authors have argued in support of postoperative radiation to prevent local recurrence, ${ }^{24}$ whereas others have reported concern for secondary risks of irradiation induced malignant transformation. ${ }^{25}$ It remains to be clear if radiotherapy is of benefit in patients with subtotal resection or curettage. In our literature review, five patients received radiotherapy. Dosing ranged from 50 to $68 \mathrm{~Gy}$. In two cases, the patient also received proton therapy, 59 CGE with 45 Gy. No dosing recommendations are available for CMF, but Feuvret el al proposed 55 to $60 \mathrm{~Gy}$, consistent with other benign tumors. ${ }^{26}$ Feuvret et al believe that radiotherapy should be part of standard treatment and proposed a treatment flow diagram 
for skull CMFs. Recommendations for radiation include patients who are unresectable or received curettage or partial resection in vital neurologic areas, or have a recurrent tumor. ${ }^{26}$ In our literature review, four of the five patients who received radiation had recurrence postradiation. All of the patients who received radiotherapy had an initial subtotal resection. Though efficacy of radiotherapy in the setting of subtotal cranial CMF resection cannot be dismissed, our review did not show a benefit.

One of our patients and three patients identified in our literature review had symptom onset and subsequent diagnosis during pregnancy. This raises the possibility that these tumors are hormonally sensitive. To our knowledge, however, there is only one reported case where a hormonal influence was potentially associated with $\mathrm{CMF}^{27}$ Cytogenetic analysis of a scapular CMF revealed mutation in the parathyroid hormone/parathyroid hormone-related peptide receptor gene (PTH/PTHrP). Halbert et al indicate that this suggests the existence of autocrine/paracrine regulatory loops thought to be essential for normal chondrocyte maturation and/or endochondral bone formation.

\section{Conclusion}

Cranial CMF is rare, and consequently frequently misdiagnosed. Key features of diagnosis are radiographic and histologic findings. It is important to keep CMF in the differential diagnosis when evaluating solitary cranial bone lesions, as CMF is curable by total excision. Total resection is the best treatment, and should be the goal of any surgical intervention. Curettage results in high recurrence rates. Radiotherapy in the setting of subtotal resection or recurrence cannot be definitively recommended and needs further investigation.

\section{Acknowledgments}

Thanks to Dima Suki and Lamonne Crutcher for their help obtaining Institutional Review Board approval for this manuscript.

\section{References}

1 Rahimi A, Beabout JW, Ivins JC, Dahlin DC. Chondromyxoid fibroma: a clinicopathologic study of 76 cases. Cancer 1972;30(3):726-736

2 Jaffe HL, Lichtenstein L. Chondromyxoid fibroma of bone: a distinctive benign tumor likely to be mistaken especially for chondrosarcoma. Arch Pathol (Chic) 1948;45(4):541-551

3 World Health Organization Fletcher CDM. WHO classification of tumours of soft tissue and bone: [this book reflects the views of a working group that convened for a consensus and editorial meeting at the University of Zurich, Switzerland, 18-20 April 2012]. Internet. Agency for Research on Cancer; 2013

4 Wu CT, Inwards CY, O'Laughlin S, Rock MG, Beabout JW, Unni KK. Chondromyxoid fibroma of bone: a clinicopathologic review of 278 cases. Hum Pathol 1998;29(5):438-446

5 Kadom N, Rushing EJ, Yaun A, Santi M. Chondromyxoid fibroma of the frontal bone in a teenager. Pediatr Radiol 2009;39(1):53-56

$6 \mathrm{Xu} \mathrm{H}$, Qin Z, Shi Z. Chondromyxoid fibroma in the sella turcica region. J Clin Neurosci 2011;18(10):1419-1421
7 Zillmer DA, Dorfman HD. Chondromyxoid fibroma of bone: thirtysix cases with clinicopathologic correlation. Hum Pathol 1989; 20(10):952-964

8 Thompson AL, Bharatha A, Aviv RI, et al. Chondromyxoid fibroma of the mastoid facial nerve canal mimicking a facial nerve schwannoma. Laryngoscope 2009;119(7):1380-1383

9 Vernon SE, Casiano RR. Sphenoid sinus chondromyxoid fibroma mimicking a mucocele. Am J Otolaryngol 2006;27(6):406-408

10 Patino-Cordoba JI, Turner J, McCarthy SW, Fagan P. Chondromyxoid fibroma of the skull base. Otolaryngol Head Neck Surg 1998; 118(3 Pt 1):415-418

11 Viswanathan R, Jegathraman AR, Ganapathy K, Bharati AS, Govindan R. Parasellar chondromyxofibroma with ipsilateral total internal carotid artery occlusion. Surg Neurol 1987;28(2):141-144

12 Ozek E, Iplikcioglu AC. Chondromyxoid fibroma of the skull base: a case report of an unusual location. Cent Eur Neurosurg 2011; 72(3):152-154

13 Nazeer T, Ro JY, Varma DG, de la Hermosa JR, Ayala AG. Chondromyxoid fibroma of paranasal sinuses: report of two cases presenting with nasal obstruction. Skeletal Radiol 1996;25(8): 779-782

14 Mendoza M, González I, Aperribay M, Hermosa JR, Nogués A. Congenital chondromyxoid fibroma of the ethmoid: case report. Pediatr Radiol 1998;28(5):339-341

15 Veras EFT, Santamaria IB, Luna MA. Sinonasal chondromyxoid fibroma. Ann Diagn Pathol 2009;13(1):41-46

16 Maruyama R, Nagaoka S, Todaka T, Nakahara T, Kishida K. Intracranial chondromyxoid fibroma extending into the jugular foramen. Pathol Int 1994;44(12):857-859

17 Nielsen GP, Keel SB, Dickersin GR, Selig MK, Bhan AK, Rosenberg AE. Chondromyxoid fibroma: a tumor showing myofibroblastic, myochondroblastic, and chondrocytic differentiation. Mod Pathol 1999;12(5):514-517

18 Ditta LC, Qayyum S, O'Brien TF, Choudhri AF, Wilson MW. Chondromyxoid fibroma of the orbit. Ophthal Plast Reconstr Surg 2012; 28(5):e105-e106

19 Morris LGT, Rihani J, Lebowitz RA, Wang BY. Chondromyxoid fibroma of sphenoid sinus with unusual calcifications: case report with literature review. Head Neck Pathol 2009;3(2):169-173

20 Desai SS, Jambhekar NA, Samanthray S, Merchant NH, Puri A, Agarwal M. Chondromyxoid fibromas: a study of 10 cases. J Surg Oncol 2005;89(1):28-31

21 Khalatbari MR, Hamidi M, Moharamzad Y. Chondromyxoid fibroma of the anterior skull base invading the orbit in a pediatric patient: case report and review of the literature. Neuropediatrics 2012;43(3):140-145

22 Shek TW, Peh WC, Leung G. Chondromyxoid fibroma of skull base: a tumour prone to local recurrence. J Laryngol Otol 1999;113(4): 380-385

23 Smith CA, Magenis RE, Himoe E, Smith C, Mansoor A. Chondromyxoid fibroma of the nasal cavity with an interstitial insertion between chromosomes 6 and 19. Cancer Genet Cytogenet 2006; 171(2):97-100

24 Keel SB, Bhan AK, Liebsch NJ, Rosenberg AE. Chondromyxoid fibroma of the skull base: a tumor which may be confused with chordoma and chondrosarcoma. A report of three cases and review of the literature. Am J Surg Pathol 1997;21(5):577-582

25 LeMay DR, Sun JK, Mendel E, Hinton DR, Giannotta SL. Chondromyxoid fibroma of the temporal bone. Surg Neurol 1997;48(2): 148-152

26 Feuvret L, Noël G, Calugaru V, Terrier P, Habrand J-L. Chondromyxoid fibroma of the skull base: differential diagnosis and radiotherapy: two case reports and a review of the literature. Acta Oncol 2005;44(6):545-553

27 Halbert AR, Harrison WR, Hicks MJ, Davino N, Cooley LD. Cytogenetic analysis of a scapular chondromyxoid fibroma. Cancer Genet Cytogenet 1998;104(1):52-56 